\title{
KRAS G12V Mutation in Acquired Resistance to Combined BRAF and MEK Inhibition in Papillary Thyroid Cancer
}

Dwight H. Owen, MD, MSa; Bhavana Konda, MDa; Jennifer Sipos, MD; Tom Liu, PhDc; Amy Webb, PhDd; Matthew D. Ringel, MDº Cynthia D. Timmers, $\mathrm{PhD}^{\mathrm{c}, \mathrm{e}, \star}$; and Manisha H. Shah, MD ${ }^{a, \star}$

\begin{abstract}
BRAF V600E mutations occur in approximately $40 \%$ of all patients with papillary thyroid cancer (PTC) and are associated with a worse prognosis in population studies. Treatment with single-agent BRAF inhibitors can result in nondurable partial responses (PRs) in clinical trials, but resistance inevitably develops. The mechanisms of resistance are not completely understood, but in non-thyroid tumors harboring BRAF V600E mutations, resistance has been ascribed to concurrent or acquired mutations in MEK1/2, RAC1, KRAS, and NRAS. This case report describes a patient with radioactive iodine-refractory metastatic PTC treated in a clinical trial with combination BRAF and MEK inhibition who achieved a durable PR. At time of progression, biopsy revealed an acquired KRAS G12V-activating mutation. The patient subsequently went on to have a PR to cabozantinib therapy in the clinical trial. This is the first reported case of an acquired KRAS-activating mutation that developed during treatment with BRAF and MEK inhibition in a patient with BRAFmutated PTC. The KRAS mutation was also detected in peripheral blood samples taken as part of the trial, indicating that resistant mutations may be identified through noninvasive means. The identification of resistant mutations in patients at time of progression is necessary to identify possible therapeutic options including potential clinical trials. ClinicalTrials.gov identifier: NCT01723202
\end{abstract}

J Natl Compr Canc Netw 2019;17(5):409-413 doi: 10.6004/jnccn.2019.7292

${ }^{a}$ Division of Medical Oncology, ${ }^{b}$ Division of Endocrinology, Diabetes, and Metabolism, 'Solid Tumor Translational Service, and dDepartment of Biomedical Information, The Ohio State University Wexner Medical Center and Comprehensive Cancer Center, Columbus, Ohio; and ${ }^{\mathrm{e} M e d i c a l}$ University of South Carolina, Charleston, South Carolina.
Over the past several years, clinical trials have led to FDA approval of the multikinase inhibitors (MKI) lenvatinib (February 2015) ${ }^{1}$ and sorafenib (November 2013) ${ }^{2}$ for treatment of radioactive iodine (RAI)-refractory, progressive, differentiated thyroid cancer (DTC). Yet, for both of these MKIs, acquired resistance is universal, adverse events are common, and no overall survival benefit has been demonstrated. Papillary thyroid cancer (PTC) is primarily driven by constitutive activation of the RAS/ RAF/MEK/ERK pathway, a key oncogenic signaling cascade for many human malignancies. ${ }^{3}$ Activating $B R A F$ mutations are the most common cause for this activation in PTC, occurring in $25 \%$ to $49 \%$ of tumors. Moreover, the presence of this mutation is associated with more advanced disease and poorer prognosis. ${ }^{4-6}$ Although there are currently no approved BRAF-targeted treatments for patients with PTC, a phase II trial of the BRAF inhibitor vemurafenib in patients with RAI-refractory, BRAF-mutated PTC demonstrated a response rate of $35 \% .^{7}$ Resistance to BRAF inhibition is likely to develop eventually, which has been demonstrated in melanoma, and is thought to occur through reactivation of the MAPK pathway. ${ }^{8}$

Combination dabrafenib/trametinib is now the standard therapy for patients with melanoma harboring $B R A F$ V600E mutations based on increased response rates and overall survival. ${ }^{9}$ However, resistance to dual inhibition eventually develops in most patients due to somatic mutations in MEK1/2, KRAS, or NRAS, and amplification of the $B R A F$ V600E mutant alleles. ${ }^{10-13}$ Mechanisms of resistance to combination BRAF and MEK inhibition remain to be fully elucidated in PTC. Danysh et $\mathrm{al}^{14}$ reported in vitro studies wherein a $B R A F$ V600E-mutated thyroid cancer cell line selected for resistance to vemurafenib developed an acquired novel KRAS G12D-activating mutation. Cabanillas et $\mathrm{al}^{15}$ reported a case of a patient with anaplastic thyroid carcinoma treated with dabrafenib/trametinib in whom an

See JNCCN.org for supplemental online content.

*These authors contributed equally. 
NRAS Q61K mutation was discovered on tumor tissue after 4 weeks of treatment. The present case report describes for the first time the development of an activating KRAS G12V mutation as a potential resistance mechanism in a patient with PTC treated with combination dabrafenib/trametinib who experienced a subsequent response to cabozantinib.

\section{Case Report}

A 67-year-old woman diagnosed with PTC underwent total thyroidectomy with central neck dissection, which revealed a $7.2-\mathrm{cm}$ extensive right lobar, poorly differentiated PTC with 3 of 9 lymph nodes positive and a background of Hashimoto thyroiditis. Following surgical resection, imaging revealed bilateral pulmonary nodules and mediastinal adenopathy. She received $100.9 \mathrm{mCi}$ of RAI therapy, and a posttreatment scan showed uptake in the thyroid bed but none in the chest. The tumor was staged as a pT3pN1aM0 poorly DTC. Repeat imaging 6 months after initial diagnosis and treatment revealed increasing adenopathy in the neck and bilateral subcentimeter pulmonary nodules, and the patient underwent right radical neck dissection with 4 of 52 examined lymph nodes positive for PTC, with no extranodal extension noted. Six months later, imaging again revealed an enlarged right paratracheal node and anterior paratracheal node, which were resected and determined to be positive for PTC. The patient went on to receive external-beam radiotherapy to the neck at an outside institution. She was then started on sorafenib, $400 \mathrm{mg}$ twice daily, which initially was poorly tolerated and she developed hand-foot syndrome and diarrhea. With the aid of supportive measures and minimal dose interruption, she was able to remain on sorafenib at $400 \mathrm{mg}$ twice daily for 2 years with stable disease as the best response (supplemental eFigure 1, available with this article at JNCCN.org).

After experiencing progressive disease, the patient was enrolled in a clinical trial and started on lenvatinib (ClinicalTrials.gov identifier: NCT01321554). ${ }^{1}$ She remained on lenvatinib, $24 \mathrm{mg}$ daily, for 6 months before experiencing progressive disease in both the neck and chest. Her treatment course was complicated by hypertension, hand-foot syndrome, hypercalcemia, and proteinuria, requiring dose interruptions and reductions. Her tissue from previously resected metastatic lymph nodes was assessed by PCR for the presence of a $B R A F$ mutation and found to be positive for BRAF V600E. She was then enrolled in another clinical trial (NCT01723202) and randomly assigned to combination therapy with dabrafenib, $150 \mathrm{mg}$ twice daily, and trametinib, $2 \mathrm{mg}$ daily. After 2 cycles, she sustained a partial response (PR) in the thyroid bed, cervical and intrathoracic lymph nodes, and pulmonary lesions, with a RECIST v1.1 decrease of $67 \%$ in the target lesions, including a dramatic decrease in the size of the thyroid bed tumor from 6.1 to $2.3 \mathrm{~cm}$ (Figure 1). Thyroglobulin levels initially increased from $1,601 \mathrm{ng} / \mathrm{mL}$ at baseline to $3,620 \mathrm{ng} / \mathrm{mL}$ after 2 months of therapy, before decreasing steadily to a nadir of $385 \mathrm{ng} / \mathrm{mL}$ at the time of disease progression. The patient's treatment course was complicated by rash and fevers, which required low doses of prednisone and reduced doses of dabrafenib (100 mg orally twice daily) and trametinib (1.5 mg daily). She also experienced an episode of cholecystitis that was treated with cholecystectomy after 8 cycles, but otherwise experienced minimal toxicities.

The patient was maintained on combination therapy for 18 months before discontinuing the trial due to progressive disease. A biopsy was obtained at progression and whole-exome sequencing was performed on the biopsy tissue, her germline DNA, and DNA purified from the archival primary tumor (eAppendix 1). BRAF V600E mutation was verified in both the archival and progression tumor biopsies, in addition to 24 other deleterious mutations found in the original tumor. Mutations detected at progression were cross-referenced against Condel, SIFT, PolyPhen, and PROVEAN and were considered a candidate if they were identified as deleterious or damaging on at least 3 of the 4 platforms (supplemental eTables 1-3). Of these mutations, the KRAS G12V mutation was most likely the driver of resistance to therapy. In addition, blood-based digital droplet PCR (ddPCR) was performed as part of the clinical study (eAppendix 1), which demonstrated both a rapid decline in $B R A F$ V600E copies after therapy initiation and a reemergence of BRAF $\mathrm{V} 600 \mathrm{E}$ in addition to KRAS G12V at clinical progression (Figure 2). Both BRAF and KRAS ddPCR were detectable 2 cycles before treatment discontinuation due to clinical progression. The patient subsequently received cabozantinib therapy on a clinical trial (ClinicalTrials.gov identifier: NCT01811212) and experienced a PR, with a $45 \%$ reduction in target lesions. She continued on therapy for 9 months at a reduced dose due to hand-foot syndrome and hyponatremia, before experiencing a decline in performance status and progressive disease, and died soon thereafter.

\section{Discussion}

Thyroid cancer is the most common form of endocrine malignancy worldwide, and DTC is the most common histologic subtype, which includes papillary, follicular, and Hürthle cell histologies. Treatment is typically surgery, and in select cases is followed by RAI and thyroidstimulating hormone suppression therapy. For patients who develop metastatic RAI-refractory disease, treatment options are limited. Recently, the 2 MKIs sorafenib and levatinib were FDA-approved for this population based on large phase III trials, yet neither have demonstrated 


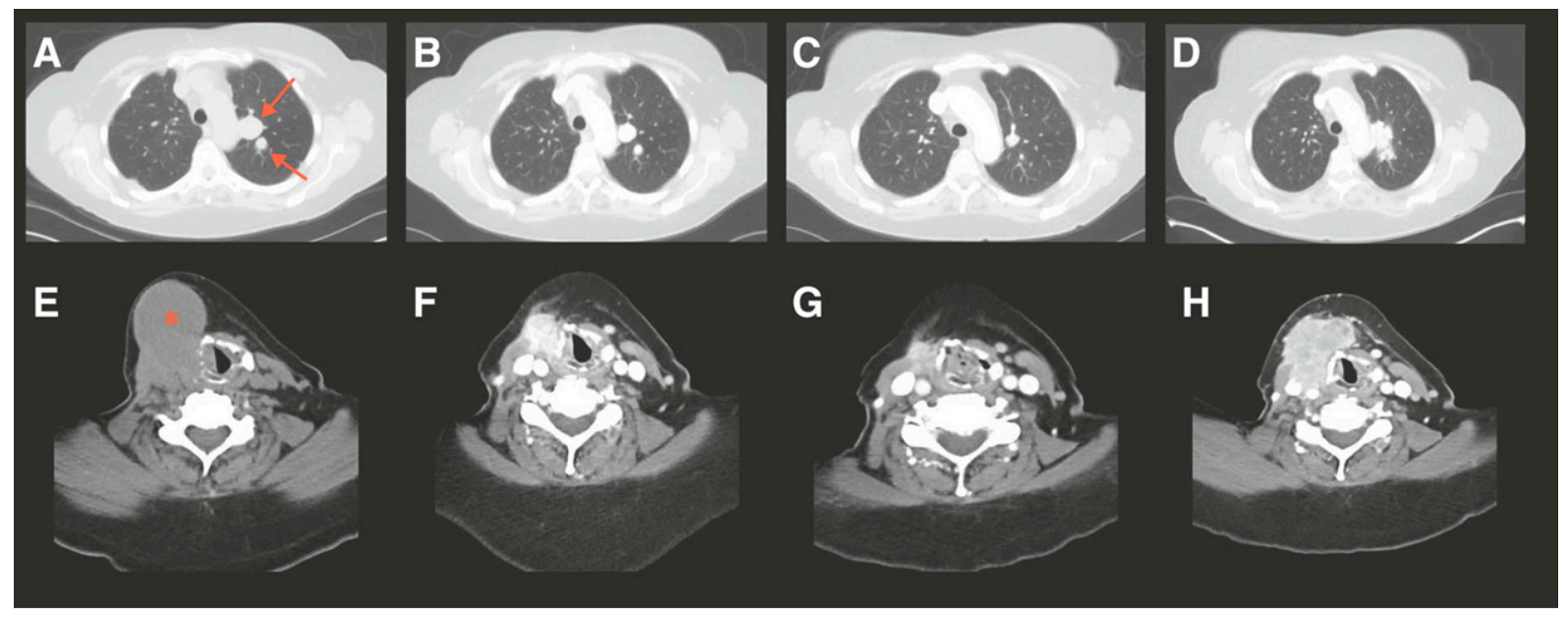

Figure 1. Clinical response to combined BRAF and MEK inhibition. Representative images are from chest CT (A-D) and neck CT (E-H) of the target lesions in the left upper lobe (arrows) and right thyroid bed (asterisk) at baseline ( $A, E)$, after 2 cycles (B, F), after 10 cycles (C, G), and at progressive disease after 20 cycles (D, H).

complete remission or improved survival. ${ }^{1,2}$ As our aforementioned experience demonstrates, these medications are associated with significant toxicities, including hypertension, hand-foot syndrome, fatigue, nausea, diarrhea, bleeding, thrombosis, and cardiac arrhythmias. ${ }^{16}$ Oncogenic mutations in BRAF V600E occur in roughly half of patients with PTC and are associated with poor prognosis. ${ }^{6}$ We and others ${ }^{17}$ have demonstrated that clinical trial populations are enriched for patients with this mutation. Clinical trials are ongoing with BRAF inhibitors, including a recently completed phase II trial of vemurafenib in 51 patients, which demonstrated an overall response rate of $35 \% .^{7}$ Based on studies in patients with melanoma in which combined BRAF and MEK inhibition was observed to improve response and survival, ${ }^{18}$ a clinical trial of dabrafenib alone or in

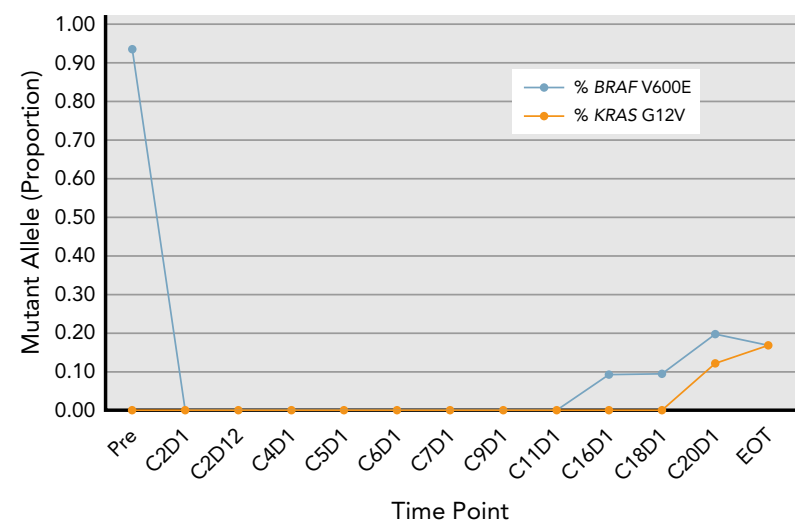

Figure 2. Blood-based monitoring of BRAF V600E and KRAS G12V mutations demonstrate possible emergence of resistance that corresponded to clinical disease progression.

Abbreviation: EOT, end of treatment. combination with trametinib in patients with RAIrefractory, $B R A F$-mutated thyroid cancer is underway (ClinicalTrials.gov identifier: NCT01723202). Furthermore, preclinical and clinical studies of BRAF and/or MEK inhibitors in early-stage, RAI-refractory DTC show promising new avenues for treatment of these patients; this is based on the ability of BRAF and/or MEK inhibitors to stimulate RAI uptake, thereby regaining iodine avidity that can be targeted by RAI. Such therapy may be more suitable for patients with low tumor burden and indolent disease before FDA-approved MKIs are considered. ${ }^{19-22}$

As with other targeted agents, resistance eventually develops in patients treated with combination BRAF and MEK inhibitors. Previously described mechanisms of resistance to BRAF inhibition in thyroid cancer have been focused on primary resistance and included alternate $B R A F$ splicing, ${ }^{23} \mathrm{c}$-MET-mediated reactivation of the PI3K/AKT pathway, ${ }^{24}$ and copy number gain of MCL1 and loss of CDKN2A. ${ }^{25}$ Potential resistance mechanisms to BRAF and/or MEK inhibition, described in other solid tumors, include mutations in MEK1/2, RAC1, KRAS, or $N R A S$, and amplification of the BRAF V600E mutant alleles. ${ }^{11,12} K R A S, A R A F$, and MEK1-resistance mutations have been described in patients with $B R A F$-mutated colon cancer treated with combination dabrafenib/ trametinib. ${ }^{10,13}$ Recently, a study found that prolonged treatment of thyroid cancer cell lines with vemurafenib led to the development of a KRAS G12D mutation, which the investigators proposed may confer resistance by sustaining RAS/MEK/ERK signaling and PI3K/AKT pathway activation through EGFR and HER3. ${ }^{14}$ Importantly, although these cells were resistant to vemurafenib monotherapy, combination treatment of vemurafenib and PI3K and ERK1/2 inhibitors remained active, whereas BRAF 
and MEK inhibition did not. This is further supported by the recent report of an NRAS Q61K mutation seen in a patient with anaplastic thyroid cancer after 4 weeks of treatment with combination BRAF and MEK inhibition. ${ }^{15}$

By the time our patient began BRAF and MEK combination therapy, she had exhausted all standard options for PTC, including surgical resection, RAI, external-beam radiotherapy, and both FDA-approved MKIs sorafenib and lenvatinib. She had significant tumor burden in her neck, chest, and bones. Despite this, she experienced a sustained PR to treatment with BRAF and MEK inhibition for 18 months with improvement in her tumor burden. Our patient's trend in serum thyroglobulin levels is also of high interest, with an initial uptrend despite PR and levels decreasing to nadir at progression. This is possibly secondary to tumor redifferentiation that can be seen with BRAF inhibitor therapy. ${ }^{19,26,27}$ The finding of a KRAS G12V mutation supports the preclinical models of resistance to single-agent BRAF inhibition in thyroid cancer and combination BRAF and MEK inhibition in both melanoma and colon cancer. ${ }^{10,13,14}$ For example, preclinical studies in colorectal cancer have revealed that KRAS mutations in G12D and G13D led to resistance to combined BRAF and MEK inhibition, and in a clinical study of dabrafenib, trametinib, and panitumumab in patients with $B R A F-m u-$ tated colon cancer, KRAS or NRAS mutations were detected at the time of disease progression but not at baseline. ${ }^{10,13}$ A second preclinical study of colorectal cancer found acquired KRAS mutations in exons 2 and 4 (G12D, G13D, and A146T/V) to combination therapies with BRAF and EGFR inhibition or combination BRAF, EGFR, and PI3K- $\alpha$ inhibitors, while the same authors reported the emergence of an acquired mutation in KRAS G12C in a patient with colorectal cancer treated with combination BRAF and MEK inhibition in a clinical trial. ${ }^{28} \mathrm{In}$ melanoma, mutations in NRAS and KRAS have been reported in patients who developed resistance to BRAF inhibitors, but NRAS mutations were far more common $(17 \%$ vs $2 \%$ ), and specific allelic mutations were not reported. ${ }^{29}$

A limitation of our study is that the archival tumor specimen was obtained at the time of surgical reresection before treatment with external-beam radiotherapy, sorafenib, and lenvatinib. Ideally, both tissue and cell-free DNA would be evaluated at baseline before BRAF and MEK inhibition to ensure that the KRAS mutation did not develop as a result of prior treatment, although the absence of KRAS on cell-free DNA at baseline is reassuring. Further research is needed to confirm this resistance mechanism, and to develop strategies to both prevent resistance and prolong clinical responses, such as combination therapy with downstream ERK inhibitors. In preclinical models, MEK-resistant cell lines retained sensitivity to selective ERK1/2 inhibition in colorectal cell lines. ${ }^{30}$ Clinical trials with ERK inhibitors are ongoing with dose escalation in both a singleagent (ClinicalTrials.gov identifier: NCT01781429) and in combination with chemotherapy (NCT02608229). Our patient's subsequent response to cabozantinib indicates the importance of identifying the optimal sequencing strategy for patients who develop resistance to targeted therapy. In a subset analysis of a phase III trial of cabozantinib in medullary thyroid cancer, patients with $R A S$ mutations seemed to derive clinical benefit in terms of response rates and progression-free survival. ${ }^{31}$ The mechanism through which cabozantinib may exert its effect on RAS-mutated tumors remains unclear, but preclinical models suggest that MET signaling may be essential for KRAS-mediated, anchorage-independent cell growth. ${ }^{32}$ MET inhibition by cabozantinib may impact downstream ERK and AKT and may therefore have contributed to the clinical response seen in our patient. Finally, the clinical responses observed by Iyer et $\mathrm{al}^{33}$ in patients with resistant anaplastic thyroid cancer treated with pembrolizumab and targeted therapy indicates the possible role of checkpoint inhibitors in this setting.

\section{Conclusions}

This report presents a patient with $B R A F$-mutated PTC who initially sustained an excellent response to treatment with BRAF and MEK inhibition but was found to have developed a KRAS G12V mutation at time of progression that may be a secondary resistance mechanism. This observation is further supported by data from peripheral blood ddPCR showing a decline in BRAF V600E detection during treatment, followed by the redetection of both BRAF V600E and KRAS G12V mutations at the time of clinical progression. Further research, including prospective clinical trials, should include assessment of $B R A F$ $\mathrm{V} 600 \mathrm{E}$ at the time of disease progression both within the tumor and from blood-based assays. Finally, strategies to prevent the development of resistance should be explored. For instance, our patient's subsequent response to cabozantinib may implicate AKT and ERK as viable therapeutic targets, as suggested in preclinical studies. In addition, combination or sequential treatment with immune checkpoint inhibitors may abrogate the development of resistance.

\section{Acknowledgments}

Clinical study NCT01723202 was approved and funded in part by the NCCN Oncology Research Program, with general research support provided by Novartis. NCT01811212 was sponsored by the NCI Cancer Therapy Evaluation Program and the International Thyroid Oncology Group (ITOG). NCT01321554 was sponsored by Eisai Inc.

Submitted August 29, 2018; accepted for publication March 4, 2019

Author contributions: Study concept and design: Owen, Konda, Sipos, Ringel, Timmers, Shah. Data acquisition: Owen, Konda, Sipos, Liu, Ringel, Timmers, 
Shah. Data analysis and interpretation: All authors. Drafting of manuscript: Owen, Konda, Ringel, Timmers, Shah. Critical revision: All authors. Final approval: All authors.

Disclosures: Dr. Shah has disclosed that she is a scientific advisor for Novartis and receives grant/research support from Eisai Inc. The remaining authors have not received any financial consideration from any person or organization to support the preparation, analysis, results, or discussion of this article.
Funding: Clinical study NCT01723202 was approved and funded in part by the NCCN Oncology Research Program (NCCNGSK20008). Support for this research was provided by The Ohio State University Comprehensive Cancer Center using Pelotonia funds.

Correspondence: Dwight H. Owen, MD, MS, Division of Medical Oncology، The Ohio State University Wexner Medical Center and Comprehensive Cancer Center, 320 West 10th Avenue, A450B Starling Loving Hall, Columbus, OH 43210. Email: Dwight.owen@osumc.edu

\section{References}

1. Schlumberger $M$, Tahara $M$, Wirth $L J$, et al. Lenvatinib versus placebo in radioiodine-refractory thyroid cancer. N Engl J Med 2015;372:621-630.

2. Brose MS, Nutting CM, Jarzab B, et al. Sorafenib in radioactive iodinerefractory, locally advanced or metastatic differentiated thyroid cancer: a randomised, double-blind, phase 3 trial. Lancet 2014;384: 319-328.

3. McCubrey JA, Steelman LS, Chappell WH, et al. Roles of the Raf/MEK/ ERK pathway in cell growth, malignant transformation and drug resistance. Biochim Biophys Acta 2007;1773:1263-1284.

4. Brzezianska E, Pastuszak-Lewandoska D. A minireview: the role of MAPK/ ERK and PI3K/Akt pathways in thyroid follicular cell-derived neoplasm. Front Biosci 2011;16:422-439.

5. Xing M. Prognostic utility of BRAF mutation in papillary thyroid cancer. Mol Cell Endocrinol 2010;321:86-93.

6. Xing $M$, Westra $W H$, Tufano RP, et al. BRAF mutation predicts a poorer clinical prognosis for papillary thyroid cancer. J Clin Endocrinol Metab 2005:90:6373-6379.

7. Brose MS, Cabanillas ME, Cohen EE, et al. Vemurafenib in patients with BRAF(V600E)-positive metastatic or unresectable papillary thyroid cancer refractory to radioactive iodine: a non-randomised multicentre, open-label, phase 2 trial. Lancet Oncol 2016;17: 1272-1282.

8. Rizos H, Menzies AM, Pupo GM, et al. BRAF inhibitor resistance mechanisms in metastatic melanoma: spectrum and clinical impact. Clin Cancer Res 2014;20:1965-1977.

9. Long GV, Stroyakovskiy D, Gogas H, et al. Combined BRAF and MEK inhibition versus BRAF inhibition alone in melanoma. N Engl J Med 2014; 371:1877-1888.

10. Ahronian LG, Sennott EM, Van Allen EM, et al. Clinical acquired resistance to RAF inhibitor combinations in BRAF-mutant colorectal cancer through MAPK pathway alterations. Cancer Discov 2015;5:358-367.

11. Gowrishankar K, Snoyman S, Pupo GM, et al. Acquired resistance to BRAF inhibition can confer cross-resistance to combined BRAF/MEK inhibition. J Invest Dermatol 2012;132:1850-1859.

12. Wagle N, Van Allen EM, Treacy DJ, et al. MAP kinase pathway alterations in BRAF-mutant melanoma patients with acquired resistance to combined RAF/MEK inhibition. Cancer Discov 2014;4:61-68.

13. Corcoran RB, André T, Atreya CE, et al. Combined BRAF, EGFR, and MEK inhibition in patients with BRAF V600E-mutant colorectal cancer. Cancer Discov 2018;8:428-443.

14. Danysh BP, Rieger EY, Sinha DK, et al. Long-term vemurafenib treatment drives inhibitor resistance through a spontaneous KRAS G12D mutation in a BRAF V600E papillary thyroid carcinoma model. Oncotarget 2016;7: 30907-30923.

15. Cabanillas ME, Ferrarotto R, Garden AS, et al. Neoadjuvant BRAF- and immune-directed therapy for anaplastic thyroid carcinoma. Thyroid 2018; 28:945-951.

16. Cabanillas ME, Hu MI, Durand JB, et al. Challenges associated with tyrosine kinase inhibitor therapy for metastatic thyroid cancer. J Thyroid Res 2011;2011:985780.

17. Kloos RT, Ringel MD, Knopp MV, et al. Phase II trial of sorafenib in metastatic thyroid cancer. J Clin Oncol 2009;27:1675-1684.
18. Flaherty KT, Infante JR, Daud A, et al. Combined BRAF and MEK inhibition in melanoma with BRAF V600 mutations. N Engl J Med 2012;367: 1694-1703.

19. Rothenberg SM, McFadden DG, Palmer EL, et al. Redifferentiation of iodine-refractory BRAF V600E-mutant metastatic papillary thyroid cancer with dabrafenib. Clin Cancer Res 2015;21:1028-1035.

20. Cheng $L$, Jin $Y$, Liu $M$, et al. HER inhibitor promotes BRAF/MEK inhibitorinduced redifferentiation in papillary thyroid cancer harboring BRAFV600E. Oncotarget 2017;8:19843-19854.

21. Ho AL, Grewal RK, Leboeuf R, et al. Selumetinib-enhanced radioiodine uptake in advanced thyroid cancer. N Engl J Med 2013;368: 623-632.

22. Dunn LA, Sherman EJ, Baxi SS, et al. Vemurafenib redifferentiation of BRAF mutant, RAl-refractory thyroid cancers. J Clin Endocrinol Metab 2019;104:1417-1428.

23. Baitei EY, Zou M, Al-Mohanna F, et al. Aberrant BRAF splicing as an alternative mechanism for oncogenic B-Raf activation in thyroid carcinoma. J Pathol 2009;217:707-715.

24. Byeon $\mathrm{HK}, \mathrm{Na} \mathrm{HJ}$, Yang $\mathrm{YJ}$, et al. c-Met-mediated reactivation of $\mathrm{PI} 3 \mathrm{~K}$ AKT signaling contributes to insensitivity of BRAF(V600E) mutant thyroid cancer to BRAF inhibition. Mol Carcinog 2016;55:1678-1687.

25. Duquette M, Sadow PM, Husain A, et al. Metastasis-associated MCL1 and P16 copy number alterations dictate resistance to vemurafenib in a BRAF V600E patient-derived papillary thyroid carcinoma preclinical model. Oncotarget 2015;6:42445-42467.

26. Falchook GS, Millward M, Hong D, et al. BRAF inhibitor dabrafenib in patients with metastatic BRAF-mutant thyroid cancer. Thyroid 2015;25: 71-77

27. Konda $\mathrm{B}$, Shah $\mathrm{MH}$, Wei $\mathrm{L}$, et al. Evaluation of BRAFV600E levels in cellfree DNA (CFDNA) as a biomarker of response in BRAF V600E mutated radioactive iodine refractory (RAIR) differentiated thyroid cancer (DTC) treated with dabrafenib alone or in combination with trametinib. Presented at the 87th Annual Meeting of the American Thyroid Association: October 18-22, 2017; Victoria, British Columbia, Canada.

28. Oddo D, Sennott EM, Barault L, et al. Molecular landscape of acquired resistance to targeted therapy combinations in BRAF-mutant colorectal cancer. Cancer Res 2016;76:4504-4515.

29. Johnson DB, Menzies AM, Zimmer L, et al. Acquired BRAF inhibitor re sistance: a multicenter meta-analysis of the spectrum and frequencies, clinical behaviour, and phenotypic associations of resistance mechanisms. Eur J Cancer 2015;51:2792-2799.

30. Hatzivassiliou G, Liu B, O'Brien C, et al. ERK inhibition overcomes acquired resistance to MEK inhibitors. Mol Cancer Ther 2012;11:1143-1154.

31. Sherman SI, Clary DO, Elisei R, et al. Correlative analyses of RET and RAS mutations in a phase 3 trial of cabozantinib in patients with progressive metastatic medullary thyroid cancer. Cancer 2016;122:3856-3864.

32. Fujita-Sato S, Galeas J, Truitt M, et al. Enhanced MET translation and signaling sustains K-Ras-driven proliferation under anchorageindependent growth conditions. Cancer Res 2015;75:2851-2862.

33. lyer PC, Dadu R, Gule-Monroe M, et al. Salvage pembrolizumab added to kinase inhibitor therapy for the treatment of anaplastic thyroid carcinoma. J Immunother Cancer 2018;6:68. 
Supplemental online content for:

\section{KRAS G12V Mutation in Acquired Resistance to Combined BRAF and MEK Inhibition in Papillary Thyroid Cancer}

Dwight H. Owen, MD, MS; Bhavana Konda, MD; Jennifer Sipos, MD; Tom Liu, PhD; Amy Webb, PhD; Matthew D. Ringel, MD; Cynthia D. Timmers, PhD; and Manisha H. Shah, MD

J Natl Compr Canc Netw 2019;17(5):409-413

eFigure 1: Treatment Timeline From Initial Surgery

eTable 1: Candidate Mutations Detected at Time of Progression eTable 2: All Mutations Detected on Progression Tissue Sample eTable 3: Mutations Detected on Archival Tissue Specimen eAppendix 1: Supplemental Methods 


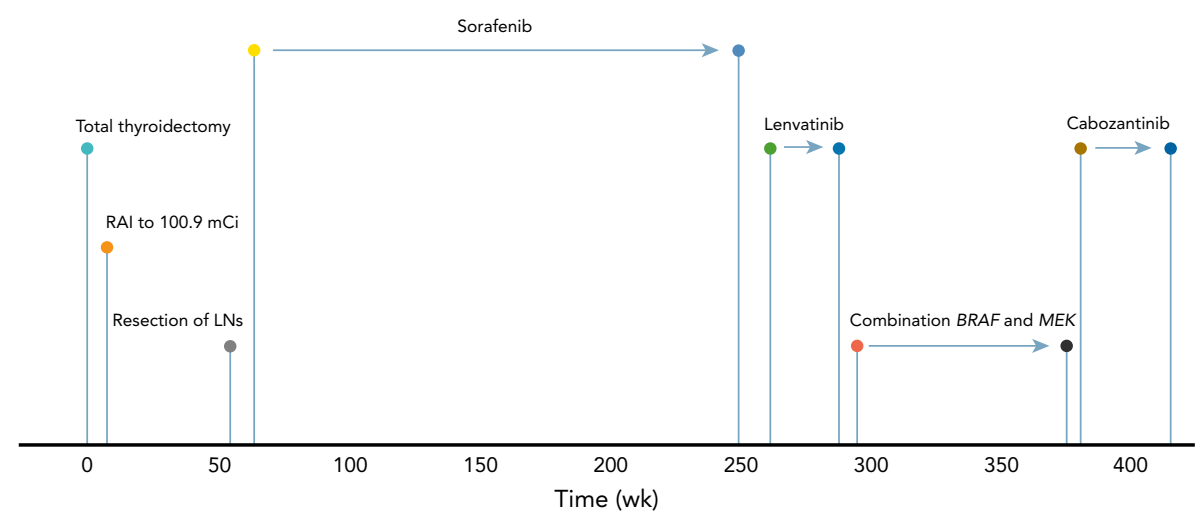

eFigure 1. Treatment timeline from initial surgery. Abbreviations: LNs, lymph nodes; RAl, radioactive iodine.

\section{eTable 1. Candidate Mutations Detected at Time of Progression}

\begin{tabular}{|llcccc}
\hline Gene & Mutation & Allelic Frequency & Consequence & Condel Score \\
\hline KRAS & G12V & 0.322 & Missense & Damaging \\
\hline UGGT1 & E13910 & 0.134 & Missense & Damaging \\
\hline RASGEF1C & A164P & 0.317 & Missense & Neutral \\
ZNF35 & S291* & 0.448 & Stop-gain & N/A \\
\hline LEUTX & S104* & 0.115 & Stop-gain & N/A \\
\hline PDHA1 & W421* & 0.522 & Stop-gain & N/A \\
\hline
\end{tabular}

Mutations detected at time of progression not present in archival specimen. Mutations detected at progression were cross-referenced with Condel, SIFT, PolyPhen, and PROVEAN and were considered a candidate if the mutation had deleterious or damaging scores on at least 3 of the 4 platforms (or stop codon). Abbreviation: N/A, not applicable. 
eTable 2. All Mutations Detected on Progression Tissue Sample

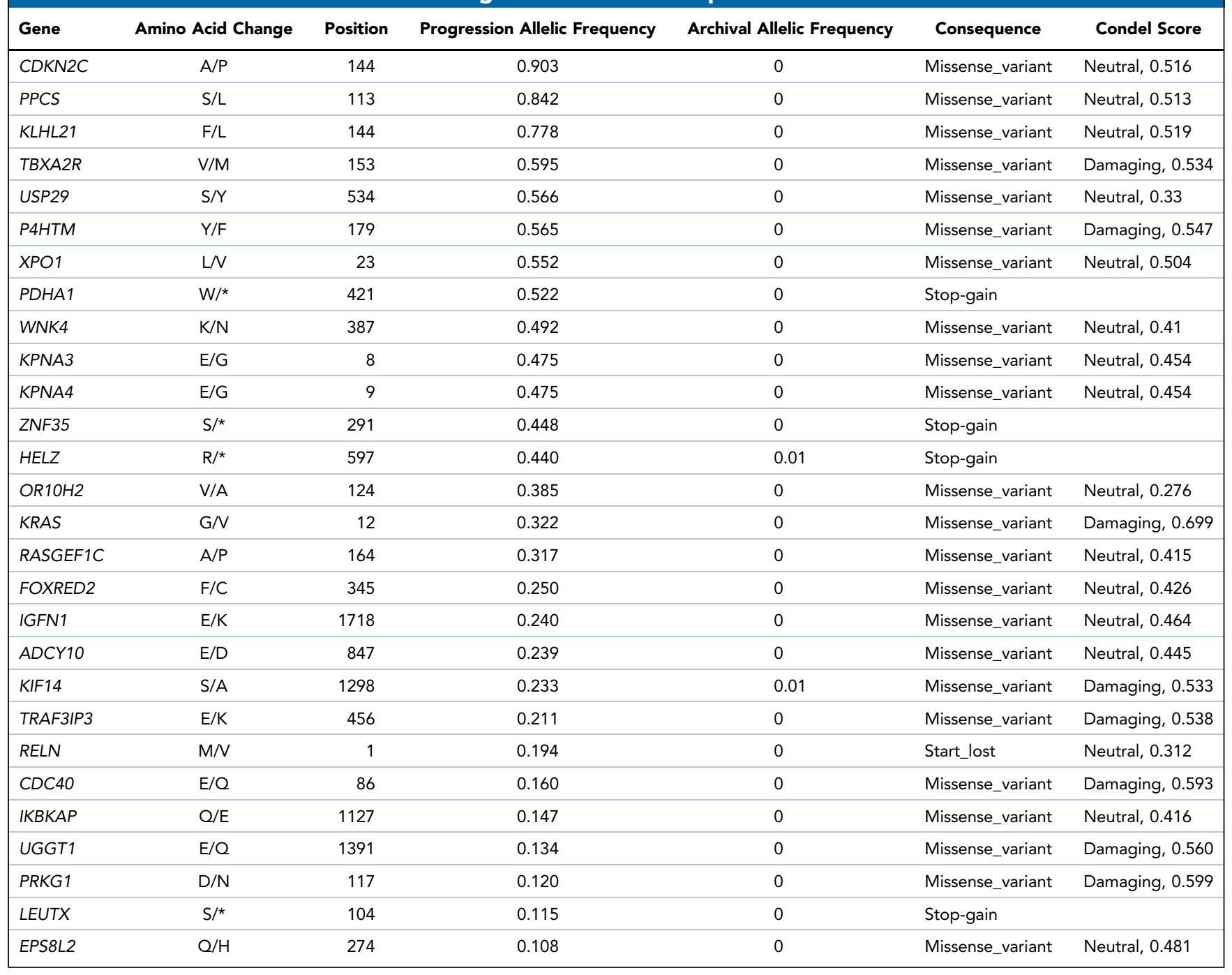




\section{eTable 3. Mutations Detected on Archival Tissue Specimen}

\begin{tabular}{|c|c|c|c|c|c|c|}
\hline Gene & Amino Acid Change & Position & $\begin{array}{l}\text { Archival Allelic } \\
\text { Frequency }\end{array}$ & $\begin{array}{c}\text { Progression Allelic } \\
\text { Frequency }\end{array}$ & Consequence & Condel Score \\
\hline POTEC & $\mathrm{A} / \mathrm{T}$ & 119 & 0.333 & 0.179 & Missense_variant & Neutral, 0.417 \\
\hline IFIT1B & $\mathrm{D} / \mathrm{N}$ & 455 & 0.129 & 0.547 & Missense_variant & Neutral, 0.416 \\
\hline ESF1 & $\mathrm{E} / \mathrm{Q}$ & 750 & 0.289 & 0.419 & Missense_variant & Neutral, 0.391 \\
\hline RBM26 & $\mathrm{F} / \mathrm{L}$ & 311 & 0.227 & 0.543 & Missense_variant & Neutral, 0.46 \\
\hline BRAF & $\mathrm{V} / \mathrm{E}$ & 600 & 0.250 & 0.467 & Missense_variant & Damaging, 0.536 \\
\hline SLC25A24 & $\mathrm{R} / \mathrm{W}$ & 6 & 0.465 & 0.750 & Missense_variant & Neutral, 0.521 \\
\hline NBPF10 & $D / G$ & 1168 & 0.075 & 0.068 & Missense_variant & Neutral, 0.304 \\
\hline KCNK3 & $\mathrm{S} / \mathrm{F}$ & 83 & 0.350 & 0.491 & Missense_variant & Neutral, 0.484 \\
\hline$B S N$ & $P / L$ & 1894 & 0.379 & 0.544 & Missense_variant & Neutral, 0.389 \\
\hline RAG1 & $\mathrm{S} / \mathrm{R}$ & 609 & 0.324 & 0.478 & Missense_variant & Damaging, 0.702 \\
\hline $\mathrm{TCHH}$ & $E / K$ & 669 & 0.160 & 0.273 & Missense_variant & Neutral, 0.356 \\
\hline NUP93 & $\mathrm{Q} / *$ & 15 & 0.265 & 0.493 & Stop-gain & $\mathrm{N} / \mathrm{A}$ \\
\hline ANKRD30B & $\mathrm{G} / \mathrm{V}$ & 494 & 0.412 & 0.424 & $\begin{array}{l}\text { Missense_variant, } \\
\text { splice_region_variant }\end{array}$ & Neutral, 0.418 \\
\hline KRBOX4/ZNF673 & $S / L$ & 148 & 0.325 & 0.423 & Missense_variant & Neutral, 0.282 \\
\hline KIAA1024 & A/N & 897 & 0.292 & 0.528 & Missense_variant & Neutral, 0.413 \\
\hline CBR4 & $M / I$ & 122 & 0.225 & 0.590 & Missense_variant & Damaging, 0.589 \\
\hline ASCC3 & $\mathrm{G} / \mathrm{S}$ & 1122 & 0.190 & 0.434 & Missense_variant & Neutral, 0.412 \\
\hline SLC45A2 & $\mathrm{P} / \mathrm{S}$ & 241 & 0.199 & 0.503 & Missense_variant & Damaging, 0.643 \\
\hline RAB12 & $\mathrm{V} / \mathrm{I}$ & 241 & 0.269 & 0.495 & Missense_variant & Neutral, 0.438 \\
\hline HEATR5B & $\mathrm{L} / \mathrm{S}$ & 1173 & 0.311 & 0.385 & Missense_variant & Damaging, 0.623 \\
\hline RSPH10B2 & $\mathrm{T} / \mathrm{M}$ & 148 & 0.321 & 0.500 & Missense_variant & Neutral, 0.503 \\
\hline
\end{tabular}

Abbreviation: N/A, not applicable. 


\section{eAppendix 1. Supplemental Methods}

The patient was treated on a clinical trial (ClinicalTrials.gov identifier: NCT01723202), which was approved by The Ohio State University Cancer Center Institutional Review Board. Tissue was extracted from the dominant cervical nodal metastasis (see Figure 1) using ultrasound guidance of a 22-gauge needle. During the trial, the same nodal metastasis was sampled before drug initiation (Figure 1E), after 2 weeks on the study drug (Figure 1F), and at the time of disease progression, before drug discontinuation (Figure 1H).

\section{Whole-Exome Next-Generation Sequencing}

For the archival tumor tissue, a hematoxylin and eosin-stained section of the block was marked by a pathologist and tumor-containing regions were macrodissected from serial sections to enrich for tumor content. DNA was extracted using the Maxwell 16 FFPE Tissue LEV DNA Purification Kit (Promega, Inc.). Germline DNA was extracted from whole blood using the Maxwell 16 Blood DNA Purification Kit, and the Maxwell 16 Tissue DNA Purification Kit was used for the fine aspirate biopsy collected at progression. DNA was quantitated using the Quant-iT PicoGreen dsDNA Assay Kit (Thermo Fisher Scientific). Whole-exome sequencing libraries were prepared using $200 \mathrm{ng}$ of genomic DNA with the SureSelectXT Reagent Kit and the SureSelectXT Clinical Research Exome capture baits (Agilent Technologies). Libraries were sequenced on an Illumina HiSeq 4000 to obtain paired-end $150 \mathrm{bp}$ reads. The average number of reads with a quality score $>30$ was 75 million for the tumor DNA samples and 25 million for the germline blood DNA, with coverage of $92 \%$ and $55 \%$ targeted bases at $>20 \times$, respectively.

Raw sequence reads were processed and aligned to the human reference genome using the Genome Analysis Toolkit (GATK) workflow. ${ }^{1}$ MuTect (v1.1.4) and VarScan 2 (v2.3.7) ${ }^{2,3}$ were used to identify tumor-specific variants for each the archival and progression samples. The Ensembl Variant Effect Predictor was used to annotate and determine functional consequences of tumor-specific variants. ${ }^{4}$ VarScan 2 was also used to identify copy number changes in tumor samples compared with matching normal samples.

\section{BRAF and KRAS Plasma Circulating Tumor DNA Assay}

Circulating cell-free DNA (ccfDNA) was purified from $4 \mathrm{~mL}$ of plasma using the Promega Maxwell HT ccfDNA Plasma Kit. Circulating tumor DNA analysis was performed by quantifying levels of the BRAFand KRAS mutations in ccfDNA using the Bio-Rad Droplet Digital PCR Mutation Detection Assays (Bio-Rad BRAFassay ID: dHsaMDV2010027; KRAS assay ID: dHsaMDV2510592) on the QX200 Droplet Digital PCR System (Bio-Rad). Droplets were analyzed with the QX200 Droplet Reader (BioRad) for fluorescent measurement of FAM and HEX probes. Gating was performed based on positive and negative controls, and mutant populations were identified. All reactions were run in duplicate. The digital droplet PCR data were analyzed with QuantaSoft Analysis Software (BioRad) to obtain fractional abundance of the mutant DNA alleles in the wild-type/normal background.

\section{References}

1. Van der Auwera GA, Carneiro MO, Hartl C, et al. From FastQ data to high confidence variant calls: the Genome Analysis Toolkit best practices pipeline. Curr Protoc Bioinformatics 2013;43:11.10.1-11.10.33.

2. KoboldtDC, Chen K, Wylie T, etal. VarScan: variant detection in massively parallel sequencing of individual and pooled samples. Bioinformatics 2009;25:2283-2285.

3. Koboldt DC, Zhang $\mathrm{Q}$, Larson DE, et al. VarScan 2: somatic mutation and copy number alteration discovery in cancer by exome sequencing. Genome Res 2012;22: 568-576.

4. McLaren W, Pritchard B, Rios D, et al. Deriving the consequences of genomic variants with the Ensembl API and SNP Effect Predictor. Bioinformatics 2010;26: 2069-2070. 